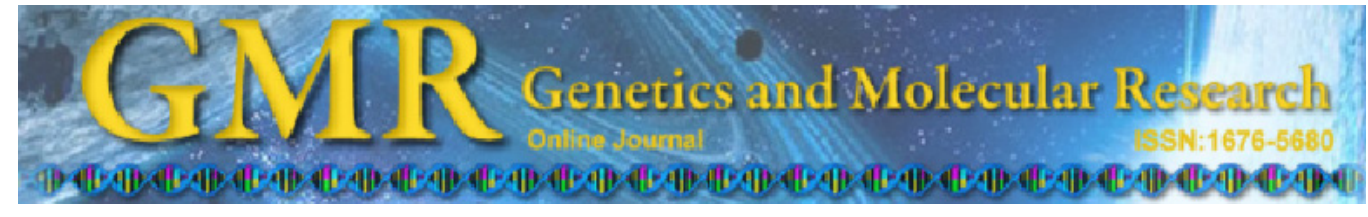

\title{
NPRL2 gene expression in the progression of colon tumors
}

\author{
B. Yogurtcu ${ }^{1}$, I. Hatemi ${ }^{2}$, I. Aydin ${ }^{3}$ and N. Buyru ${ }^{1}$ \\ ${ }^{1}$ Department of Medical Biology, Cerrahpasa Medical Faculty, \\ Istanbul University, Kocamustafapasa, Istanbul, Turkey \\ ${ }^{2}$ Department of Gastroenterology, Cerrahpasa Medical Faculty, \\ Istanbul University, Kocamustafapasa, Istanbul, Turkey \\ ${ }^{3}$ Department of General Surgery, Istanbul Training and Research Hospital, \\ Istanbul, Turkey \\ Corresponding author: N. Buyru \\ E-mail: nbuyru@yahoo.com
}

Genet. Mol. Res. (2012) Ahead of Print

Received March 29, 2012

Accepted July 31, 2012

Published September 12, 2012

DOI http://dx.doi.org/10.4238/2012.September.12.3

\begin{abstract}
Genetic and epigenetic factors affecting DNA methylation and gene expression are known to be involved in the development of colon cancer, but the full range of genetic alterations and many key genes involved in the pathogenesis of colon cancer remain to be identified. NPRL2 is a candidate tumor suppressor gene identified in the human chromosome 3 p21.3 region. We evaluated the role of this gene in the pathogenesis of colorectal cancer by investigating NPRL2 mRNA expression in 55 matched normal and tumor colon tissue samples using quantitative RT-PCR analysis. There was significantly decreased NPRL2 expression in $45 \%$ of the patients. Lower NPRL2 expression was observed significantly more frequently in poorly differentiated tumor samples than in highly or moderately differentiated tumors. We conclude that expression of NPRL2 contributes to progression of colon cancer.
\end{abstract}

Key words: Colon cancer; NPRL2; Expression; Real time-PCR; Tumor suppressor gene 
B. Yogurtcu et al.

\section{INTRODUCTION}

Colorectal cancer (CRC) is the third most common cancer, incidence worldwide is increasing, and almost $80 \%$ of cases are sporadic (Jemal et al., 2009; Ferlay et al., 2010). Genetic and environmental risk factors such as lifestyle and nutrition modulate colon cancer development (Ahmed, 2006). Although improvements in early detection and treatment have decreased mortality rates in recent years, prevention and therapy of CRC remain major public health problems. Currently, the prognosis of CRC is determined primarily by pathological parameters and the stage of disease. Early diagnosis together with treatment gives patients a chance for full recovery.

Tumor suppressor genes represent one of the main classes of cancer-associated genes, and their identification constitutes a major effort in cancer research today. Loss of heterozygosity and homozygous deletion of the chromosome $3 \mathrm{p} 21.3$ region have been reported in the early stages of various epithelial tumors, such as lung, breast, cervical, oral cavity, ovary, and kidney cancer (Kok et al., 1997; Braga et al., 1999; Lazo, 1999; Lerman and Minna, 2000; Wistuba et al., 2000). Frequent chromosome losses in this region suggest that this region may harbor tumor suppressor genes, and researchers have formed a consortium to search a tumor suppressor gene in this region (Lerman and Minna, 2000). As a result of these efforts, multiple tumor suppressor genes have been identified at the centromeric domain of 3p21.3 (LUCA region) (Lerman and Minna, 2000).

The nitrogen permease regulator like-2 gene (NPRL2) is one of the candidate tumor suppressor genes identified in this region, and genomic abnormalities in the $3 \mathrm{p} 21.3$ region are found in various human cancers (Lerman and Minna, 2000; Wistuba et al., 2000). These results suggest that the NPRL2 gene may be a tumor suppressor and that its inactivation may promote tumorigenesis. The NPRL2 gene is composed of 11 exons and encodes a 380 amino-acids protein. Multiple splice isoforms of NPRL2 are expressed in different tissue types. However, the mechanism of NPRL2-mediated tumor suppression remains unknown. Recent studies have suggested that NPRL2 is involved in DNA mismatch repair, cell cycle checkpoint signaling, and regulation of apoptosis (Lerman and Minna, 2000; Wistuba et al., 2000; Schenk et al., 2003; Li et al., 2004). Overexpression of NPRL2 in some tumor cell lines has been reported to induce apoptosis and inhibit proliferation (Schenk et al., 2003; Li et al., 2004).

The aim of this study was to determine the expression level of NPRL2 as a tumor suppressor gene in patients with colon cancer.

\section{MATERIAL AND METHODS}

Tumor and corresponding normal colon tissue samples were obtained from 55 patients undergoing surgey at the Department of General Surgery in the Istanbul Medical Faculty, Istanbul University. Of the 55 cases, $19(37.26 \%)$ were women with a mean age of 66.41 years (range 53-86 years) and $32(62.74 \%)$ were men with a mean age 60.45 years (range 36-87 years). For each sample, data on age at diagnosis, gender, clinical stage, and histological tumor type and grade were recorded. The characteristics of the patients are summarized in Table 1.

Total RNA was extracted from both tumor and non-tumor samples with an SV Total 
NPRL2 expression in colon cancer

RNA Isolation Kit (Promega, USA). cDNA was synthesized from total RNA using a RevertAid First Strand cDNA Synthesis Kit (Fermentas, Lithuania) according to the manufacturer protocol.

\section{Quantitative real-time PCR (qRT-PCR)}

The real-time PCR (RT-PCR) was carried out with a Light Cycler (Roche Molecular Biochemicals, Germany) in a total volume of $20 \mu \mathrm{L}$. A primer set specific for the translated region of NPRL2, 5'-TGGGACCCAAGATCACCTAT-3' (forward) and 5'-GCTTGTTCTGCA GCTCTGG-3' (reverse), was used. Equal amounts of cDNA, derived from $10 \mathrm{ng}$ to $5 \mu \mathrm{g}$ total RNA, was used for each PCR. PCR conditions consisted of $10 \mathrm{~min}$ at $95^{\circ} \mathrm{C}, 45$ cycles of $95^{\circ} \mathrm{C}$ for $10 \mathrm{~s}, 52^{\circ} \mathrm{C}$ for $10 \mathrm{~s}$. and $72^{\circ} \mathrm{C}$ for $10 \mathrm{~s}$. and a cooling step of $30 \mathrm{~s}$ at $40^{\circ} \mathrm{C}$. Using the GAPDH (glyceraldehyde 3-phosphate dehydrogenase) gene as a control, the relative amount of mRNA was calculated by dividing the value of NPRL2 expression by that of GAPDH for each sample. External standards were prepared by serial dilution of cDNA from normal tissue. qRT-PCR was performed at least three times for each sample, which included a no-template sample as the negative control. The local ethics committee approved the study and all patients provided their written informed consent.

\section{Statistical analysis}

We used an unpaired chi-square test to analyze the differences in NPRL2 expression levels between the colon tumors and non-cancerous colon tissues. To analyze the correlation between NPRL2 expression and clinicopathological parameters, differences in the numerical data between the two groups were evaluated using the chi-square test. Statistical analysis was performed using the PASW Statistics 18 software (SPSS 18).

\section{RESULTS}

In this study, we used tissue samples from 55 patients with colon cancer. Expression of the NPRL2 gene was analyzed by qRT-PCR. Down-regulation of mRNA expression was detected in the tumor tissue samples (Figure 1). The median expression level of NPRL2 mRNA in non-cancerous colon tissue was 1.07 , while that was in tumor tissue was 0.965 . The difference between the two groups was not significant $(\mathrm{P}=0.094)$. The level of NPRL2 mRNA expression in 25 tumor samples $(45.45 \%)$ was lower than the median. In $28(50.9 \%)$ samples, NPRL2 expression was higher than the median. NPRL2 expression was not observed in two of the tumor tissue samples (Table 1).

For statistical analysis, the patients were divided into two groups according to the NPRL2 expression level. Expression levels lower and higher than the median value were classified as low and high expression, respectively. From this analysis a significant correlation was detected between NPRL2 expression and histologic grade in the tumor tissue samples. Lower NPRL2 expression was observed more frequently in poorly differentiated tumor samples than in highly or moderately differentiated tumors $(\mathrm{P}=0.033)$. However, no significant correlation was detected between the NPRL2 expression and other clinicopathological parameters such as gender, age, grade, and histologic type $(\mathrm{P}>0.05)$. 
B. Yogurtcu et al.

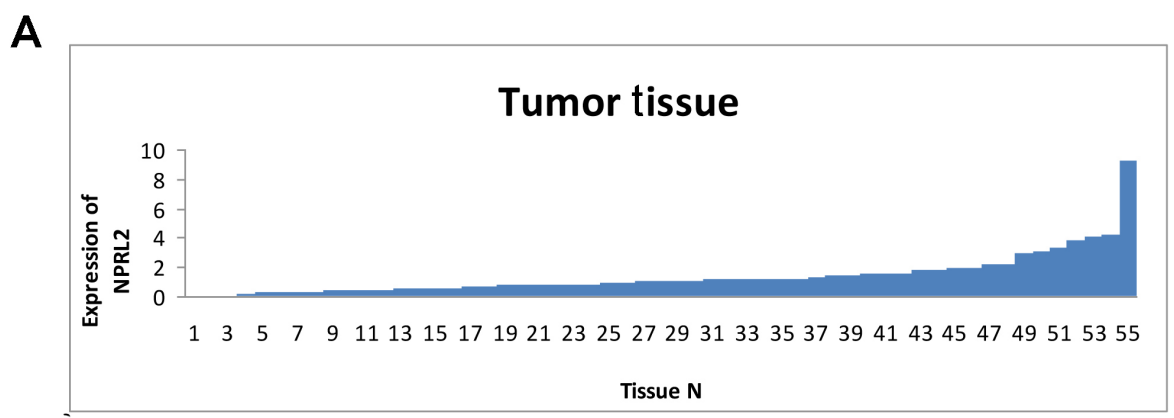

B

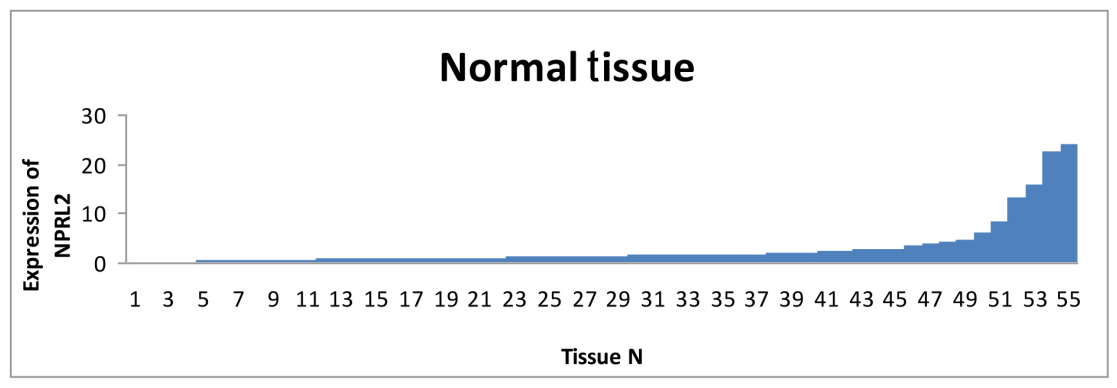

Figure 1. Expression levels of NPRL2 gene in (A) in colon tumor and (B) matched normal colon tissue samples.

\begin{tabular}{|c|c|c|c|c|c|c|c|c|c|}
\hline \multirow{2}{*}{$\begin{array}{l}\text { Clinicopathological } \\
\text { feature }\end{array}$} & \multirow[t]{2}{*}{ Variable } & \multirow{2}{*}{$\begin{array}{l}\text { Number } \\
\text { of case }\end{array}$} & \multirow[t]{2}{*}{$(\%)$} & \multicolumn{3}{|c|}{ Expression in normal tissue } & \multicolumn{3}{|c|}{ Expression in tumor tissue } \\
\hline & & & & $\begin{array}{c}\text { Increased } \\
(\%)\end{array}$ & $\begin{array}{c}\text { Unchanged } \\
(\%)\end{array}$ & $\begin{array}{c}\text { Decreased } \\
(\%)\end{array}$ & $\begin{array}{c}\text { Increased } \\
(\%)\end{array}$ & $\begin{array}{c}\text { Unchanged } \\
(\%)\end{array}$ & $\begin{array}{c}\text { Decreased } \\
(\%)\end{array}$ \\
\hline \multirow[t]{2}{*}{ Gender } & Female & 19 & 37.26 & 31.58 & 21.05 & 47.37 & 36.84 & 26.32 & 36.84 \\
\hline & Male & 32 & 62.74 & 18.75 & 31.25 & 50 & 31.25 & 40.63 & 28.12 \\
\hline \multirow[t]{2}{*}{ Age } & $60>$ & 20 & 41.7 & 40 & 25 & 35 & 25 & 40 & 35 \\
\hline & 60 ve $60<$ & 28 & 58.3 & 14.28 & 32.14 & 53.58 & 35.72 & 32.14 & 32.14 \\
\hline \multirow[t]{4}{*}{ Histologic type } & Adenocarcinoma & 33 & 68.75 & 24.24 & 27.27 & 48.48 & 36.37 & 33.33 & 30.3 \\
\hline & Mucinous & 12 & 25 & 33.3 & 33.3 & 33.3 & 25 & 41.67 & 33.33 \\
\hline & Signet ring cell & 3 & 6.25 & 0 & 33.3 & 66.7 & 0 & 25 & 75 \\
\hline & Well differentiated & 15 & 46 & 20 & 26.7 & 53.3 & 13.34 & 33.33 & 53.33 \\
\hline \multirow{3}{*}{ Histologic grade } & Moderately differentiated & 9 & 28 & 11.1 & 44.45 & 44.45 & 22.23 & 33.33 & 44.44 \\
\hline & Poorly differentiated & 8 & 25 & 50 & 25 & 25 & 37.5 & 50 & 12.5 \\
\hline & I & 2 & 6 & 0 & 50 & 50 & 0 & 50 & 50 \\
\hline \multirow[t]{3}{*}{ Stage } & II & 14 & 44 & 14.28 & 42.86 & 42.86 & 14.29 & 35.71 & 50 \\
\hline & III & 12 & 38 & 41.7 & 25 & 33.3 & 41.67 & 25 & 33.33 \\
\hline & IV & 4 & 12 & 25 & 0 & 75 & 0 & 75 & 25 \\
\hline
\end{tabular}

\section{DISCUSSION}

Loss of heterozygosity or homozygous deletion of the human chromosome $3 \mathrm{p} 21.3$ region is frequently reported in the epithelia in human cancers such as lung, breast, ovarian, cervical, head and neck, kidney, and colon tumors (Maitra et al., 2001; Imreh et al., 2003; 
Protopopov et al., 2003; Shiomi et al., 2003; Petursdottir et al., 2004; Senchenko et al., 2004). These observations have led to the identification of several tumor suppressor genes in this region (Lerman and Minna, 2000; Senchenko et al., 2004). NPRL2 is one of the candidate tumor suppressor genes mapped to the 3p21.3 region (Lerman and Minna, 2000; Wistuba et al., 2000). Recently, it has been shown that NPRL2 has tumor suppressing potential in vitro and in vivo, and it has been suggested that it is involved in DNA mismatch repair, cell cycle checkpoint signaling, and regulation of the apoptotic pathway (Ji et al., 2002; Schenk et al., 2003; Li et al., 2004). Previous reports also indicated that NPRL2 is inactivated in various human cancers and cancer cell lines by aberrant splicing of its transcripts and/or intragenic homozygous deletions (Lerman and Minna, 2000; Li et al., 2004).

Approximately $15 \%$ of all colorectal tumors are characterized by a deficiency in the DNA mismatch repair system and MSI genotype (Boland et al., 1998; Wheeler et al., 2000; Grady, 2004). Preliminary observations indicate an association between microsatellite instability and inactivating homozygous deletion of the 3'-coding region of NPRL2 in various human primary tumors ( $\mathrm{Li}$ et al., 2004). These results suggest that NPRL2 may be a novel mismatch repair gene. Therefore, we investigated NPRL2 gene expression in colon tumor tissue samples. We observed a decrease in NPRL2 mRNA level in $45.45 \%$ of the colon cancer patients. Downregulation of NPRL2 has been reported for some cancers such as non-smallcell lung carcinoma (NSCLC), hepatocellular carcinoma (HCC), and esophageal squamous cell carcinoma at different frequencies (Yi-Lo et al., 2006; Otani et al., 2009; Senchenko et al., 2010). The downregulation rate in our study is similar to the levels observed in HCC and esophageal squamous cell carcinoma (ESCC), but it is lower than the rates described for NSCLC (Yi-Lo et al., 2006; Otani et al., 2009; Senchenko et al., 2010). Senchenko et al. (2010) reported NPRL2 downregulation in 73\% of all NSCLC cases. However, downregulation of NPRL2 was significantly different between squamous cell tumors $(85 \%)$ and adenocarcinomas (44\%) in the NSCLC patients. Most of the tumors in our study group (69\%) were adenocarcinomas, and NPRL2 expression was similarly downregulated in $45.5 \%$ of this subgroup. Otani et al. (2009) reported similar NPRL2 expression levels in both normal liver and HCC tumor tissues. However, when they analyzed NPRL2 expression according to the clinicopathological characteristics of the patients, they revealed that low NPRL2 expression levels are correlated with shorter overall and disease-free survival in HCC patients (Otani et al., 2009). In our study group, the expression levels in normal and tumor tissues were different but the difference was not statistically significant. Ji et al. (2002) investigated the effect of the NPRL2 gene on tumor cell proliferation and apoptosis in human lung cancer cells using recombinant adenovirus-mediated gene transfer in vitro and in vivo. They showed that three of the candidate tumor suppressor genes located on 3p21.3, including NPRL2, significantly inhibited tumor cell growth in 3p-deficient NCI-H1299 and A549 NSCLC cells by inducing apoptosis and altering cell cycle processes. Their in vivo experiments also showed that intratumoral injection or systemic administration of Ad-NPRL2 and protamine-complexed vectors suppressed growth of $\mathrm{H} 1299$ and A549 xenografts and inhibited lung metastasis, respectively, in nu/nu mice. Our results are in accordance with these results. This is because poorly differentiated tumors in our study group. Poor differentiation is an indicator of worse prognosis with higher rates of metastasis in colon cancer (Chung et al., 1982). Ueda et al. (2006) also reported that NPRL2 might have a potential as a biomarker to predict cisplatin response and prognosis in lung cancer. They also indicated that NPRL2 might be a molecular therapeutic target for patients not responding to cisplatin. 


\section{B. Yogurtcu et al.}

In conclusion, our results indicate that a decrease in NPRL2 expression may be involved in the development of colon cancer. Therefore, the NPRL2 expression level may have utility as a prognostic factor in colon cancer. This is the first report on the NPRL2 gene expression in colon cancer demonstrating down-regulation of the gene and its association with tumor histologic grade. However, there is a need for further studies to analyze NPRL2 expression in various cancer types and larger patient groups.

\section{ACKNOWLEDGMENTS} 18806).

\section{REFERENCES}

Ahmed FE (2006). Gene-gene, gene-environment \& multiple interactions in colorectal cancer. J. Environ. Sci. Health C. Environ. Carcinog. Ecotoxicol. Rev. 24: 1-101.

Boland CR, Thibodeau SN, Hamilton SR, Sidransky D, et al. (1998). A National Cancer Institute Workshop on Microsatellite Instability for cancer detection and familial predisposition: development of international criteria for the determination of microsatellite instability in colorectal cancer. Cancer Res. 58: 5248-5257.

Braga E, Pugacheva E, Bazov I, Ermilova V, et al. (1999). Comparative allelotyping of the short arm of human chromosome 3 in epithelial tumors of four different types. FEBS Lett. 454: 215-219.

Chung CK, Zaino RJ and Stryker JA (1982). Colorectal carcinoma: evaluation of histologic grade and factors influencing prognosis. J. Surg. Oncol. 21: 143-148.

Ferlay J, Shin HR, Bray F, Forman D, et al. (2010). Estimates of worldwide burden of cancer in 2008: GLOBOCAN 2008. Int. J. Cancer 127: 2893-2917.

Grady WM (2004). Genomic instability and colon cancer. Cancer Metastasis Rev. 23: 11-27.

Imreh S, Klein G and Zabarovsky ER (2003). Search for unknown tumor-antagonizing genes. Genes Chromosomes Cancer 38: 307-321.

Jemal A, Siegel R, Ward E, Hao Y, et al. (2009). Cancer statistics, 2009. CA Cancer J. Clin. 59: 225-249.

Ji L, Nishizaki M, Gao B, Burbee D, et al. (2002). Expression of several genes in the human chromosome 3p21.3 homozygous deletion region by an adenovirus vector results in tumor suppressor activities in vitro and in vivo. Cancer Res. 62: 2715-2720.

Kok K, Naylor SL and Buys CH (1997). Deletions of the short arm of chromosome 3 in solid tumors and the search for suppressor genes. Adv. Cancer Res. 71: 27-92.

Lazo PA (1999). The molecular genetics of cervical carcinoma. Br. J. Cancer 80: 2008-2018.

Lerman MI and Minna JD (2000). The 630-kb lung cancer homozygous deletion region on human chromosome 3p21.3: identification and evaluation of the resident candidate tumor suppressor genes. The International Lung Cancer Chromosome 3p21.3 Tumor Suppressor Gene Consortium. Cancer Res. 60: 6116-6133.

Li J, Wang F, Haraldson K, Protopopov A, et al. (2004). Functional characterization of the candidate tumor suppressor gene NPRL2/G21 located in 3p21.3C. Cancer Res. 64: 6438-6443.

Maitra A, Wistuba II, Washington C, Virmani AK, et al. (2001). High-resolution chromosome 3p allelotyping of breast carcinomas and precursor lesions demonstrates frequent loss of heterozygosity and a discontinuous pattern of allele loss. Am. J. Pathol. 159: 119-130.

Otani S, Takeda S, Yamada S, Sakakima Y, et al. (2009). The tumor suppressor NPRL2 in hepatocellular carcinoma plays an important role in progression and can be served as an independent prognostic factor. J. Surg. Oncol. 100: 358-363.

Petursdottir TE, Thorsteinsdottir U, Jonasson JG, Moller PH, et al. (2004). Interstitial deletions including chromosome 3 common eliminated region 1 (C3CER1) prevail in human solid tumors from 10 different tissues. Genes Chromosomes Cancer 41: 232-242.

Protopopov A, Kashuba V, Zabarovska VI, Muravenko OV, et al. (2003). An integrated physical and gene map of the 3.5-Mb chromosome 3p21.3 (AP20) region implicated in major human epithelial malignancies. Cancer Res. 63: 404-412.

Schenk PW, Brok M, Boersma AW, Brandsma JA, et al. (2003). Anticancer drug resistance induced by disruption of the 
NPRL2 expression in colon cancer

Saccharomyces cerevisiae NPR2 gene: a novel component involved in cisplatin- and doxorubicin-provoked cell kill. Mol. Pharmacol. 64: 259-268.

Senchenko VN, Liu J, Loginov W, Bazov I, et al. (2004). Discovery of frequent homozygous deletions in chromosome 3p21.3 LUCA and AP20 regions in renal, lung and breast carcinomas. Oncogene 23: 5719-5728.

Senchenko VN, Anedchenko EA, Kondratieva TT, Krasnov GS, et al. (2010). Simultaneous down-regulation of tumor suppressor genes RBSP3/CTDSPL, NPRL2/G21 and RASSF1A in primary non-small cell lung cancer. BMC Cancer 10: 75 .

Shiomi H, Sugihara H, Kamitani S, Tokugawa T, et al. (2003). Cytogenetic heterogeneity and progression of esophageal squamous cell carcinoma. Cancer Genet. Cytogenet. 147: 50-61.

Ueda K, Kawashima H, Ohtani S, Deng WG, et al. (2006). The 3p21.3 tumor suppressor NPRL2 plays an important role in cisplatin-induced resistance in human non-small-cell lung cancer cells. Cancer Res. 66: 9682-9690.

Wheeler JM, Bodmer WF and Mortensen NJ (2000). DNA mismatch repair genes and colorectal cancer. Gut 47: 148-153.

Wistuba II, Behrens C, Virmani AK, Mele G, et al. (2000). High resolution chromosome 3p allelotyping of human lung cancer and preneoplastic/preinvasive bronchial epithelium reveals multiple, discontinuous sites of $3 p$ allele loss and three regions of frequent breakpoints. Cancer Res. 60: 1949-1960.

Yi-Lo PH, Chung Leung AC, Xiong W, Law S, et al. (2006). Expression of candidate chromosome 3p21.3 tumor suppressor genes and down-regulation of BLU in some esophageal squamous cell carcinomas. Cancer Lett. 234: 184-192. 